\title{
SINGULAR PERTURBATIONS IN OPTION PRICING
}

\author{
J.-P. FOUQUE* ${ }^{*}$ G. PAPANICOLAOU ${ }^{\dagger}$, R. SIRCAR ${ }^{\ddagger}$, AND K. SOLNA ${ }^{\S}$
}

\begin{abstract}
After the celebrated Black-Scholes formula for pricing call options under constant volatility, the need for more general nonconstant volatility models in financial mathematics has been the motivation of numerous works during the Eighties and Nineties. In particular, a lot of attention has been paid to stochastic volatility models where the volatility is randomly fluctuating driven by an additional Brownian motion. We have shown in $[2,3]$ that, in the presence of a separation of time scales, between the main observed process and the volatility driving process, asymptotic methods are very efficient in capturing the effects of random volatility in simple robust corrections to constant volatility formulas. From the point of view of partial differential equations this method corresponds to a singular perturbation analysis. The aim of this paper is to deal with the nonsmoothness of the payoff function inherent to option pricing. We present the case of call options for which the payoff function forms an angle at the strike price. This case is important since these are the typical instruments used in the calibration of pricing models. We establish the pointwise accuracy of the corrected Black-Scholes price by using an appropriate payoff regularization which is removed simultaneously as the asymptotics is performed.
\end{abstract}

1. Introduction. Stochastic volatility models in financial mathematics can be thought of as a Brownian-type particle (the stock price) moving in an environment where the diffusion coefficient is randomly fluctuating in time according to some ergodic (mean-reverting) diffusion process. We then have two Brownian motions, one driving the motion of the particle and the other driving the fluctuations of the medium. In the context of Physics there is no natural correlation between these two Brownian motions since they do not "live" in the same space. In the context of Finance they jointly define the dynamics of the stock price under its physical probability measure or an equivalent risk-neutral martingale measure. Correlation between them is perfectly natural. There are economic arguments for a negative correlation or leverage effect between stock price and volatility shocks, and from common experience and empirical studies, asset prices tend to go down when volatility goes up. The diffusion equation appears as a contingent claim pricing equation, its terminal condition being the payoff of the claim. We refer to [5] or [6] for surveys on stochastic volatility. When volatility is fast mean-reverting, on a time-scale smaller than typical maturities, one can perform a singular perturbation analysis of the pricing PDE. As we have shown in [2], this expansion reveals a first correction made of two terms: one is directly associated with the market price of volatility risk and the other is proportional to the correlation coefficient between the two Brownian motions involved. We refer to [2] for a detailed account of evidence of a fast scale in volatility and the use of this asymptotics to parametrize the evolution of the skew or the implied volatility surface. We also refer to [4] for a different type of application, namely variance reduction in Monte Carlo methods.

The present paper deals with the accuracy of such an expansion in presence of another essential characteristic feature in option pricing, namely the nonsmoothness of payoff functions. We present the case of call options since these are the liquid instruments used in the calibration of pricing models. By inverting the Black-Scholes formula the price of a call option is given in terms of its implied volatility which depends on the strike and the maturity of the option. This set of implied volatilities form the term structure of implied volatility. For fixed maturity and across strikes it is known as the smile or the skew due to the observed asymmetry. These objects and their dynamics are what volatility models are trying to reproduce in order to price and hedge other instruments.

In [2] we have performed an expansion of the price in powers of the characteristic mean-reversion time of volatility, and we have shown that the leading order term corresponds to a Black-Scholes price computed under a constant effective volatility. The first correction involves derivatives of this constant volatility price. When the payoff is smooth we have shown that the corrected price, leading order term plus first correction, has the expected accuracy, namely the remainder of the expansion is of the next order. The nonsmoothness of a call payoff which forms an angle at the strike price

\footnotetext{
*Department of Mathematics, NC State University, Raleigh NC 27695-8205, fouque@math.ncsu.edu. Work partially supported by NSF grant DMS-0071744.

${ }^{\dagger}$ Department of Mathematics, Stanford University, Stanford CA 94305,papanico@math.stanford.edu

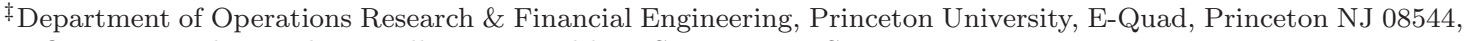
sircar@princeton.edu. Work partially supported by NSF grant DMS-0090067.

$\S$ Department of Mathematics, University of California, Irvine CA 92697, ksolna@math.uci.edu
} 
creates a singularity at the maturity time near the strike price of the option.

This paper is devoted to the proof of the accuracy of the approximation in that case. It is important because this is a natural situation in financial mathematics one has to deal with. The proof given here relies on a payoff smoothing argument which can certainly be useful in other contexts.

In Section 2 we introduce the class of stochastic volatility models which we consider. They are written directly under the pricing equivalent martingale measure and with a small parameter representing the short time-scale of volatility. We recall how option prices are given as expected values of discounted payoffs or as solutions of pricing backward parabolic PDE's with terminal conditions at maturity times. In Section 3 we recall the formal asymptotic expansion presented in [2]. In Section 4 we introduce the regularization of the payoff and decompose the main result, accuracy of the price approximation, into three Lemmas. Section 5 is devoted to the proof of these Lemmas. Detailed computations involving derivatives of Black-Scholes prices up to order seven are given in the appendices where we also recall the properties of the solutions of Poisson equations associated with the infinitesimal generator of the Ornstein-Uhlenbeck process driving the volatility.

2. Class of Models and Pricing Equations. The family of Ornstein-Uhlenbeck driven stochastic volatility models $\left(S_{t}^{\varepsilon}, Y_{t}^{\varepsilon}\right)$ that we consider can be written, under a risk-neutral probability $\mathbb{P}^{\star}$, in terms of the small parameter $\varepsilon$

$$
\begin{aligned}
d S_{t}^{\varepsilon} & =r S_{t}^{\varepsilon} d t+f\left(Y_{t}^{\varepsilon}\right) S_{t}^{\varepsilon} d W_{t}^{\star}, \\
d Y_{t}^{\varepsilon} & =\left[\frac{1}{\varepsilon}\left(m-Y_{t}^{\varepsilon}\right)-\frac{\nu \sqrt{2}}{\sqrt{\varepsilon}} \Lambda\left(Y_{t}^{\varepsilon}\right)\right] d t+\frac{\nu \sqrt{2}}{\sqrt{\varepsilon}} d \hat{Z}_{t}^{\star},
\end{aligned}
$$

where the Brownian motions $\left(W_{t}^{\star}, \hat{Z}_{t}^{\star}\right)$ have instantaneous correlation $\rho \in(-1,1)$ :

$$
d\left\langle W^{\star}, \hat{Z}^{\star}\right\rangle_{t}=\mathbb{E}^{\star}\left\{d W_{t}^{\star} d \hat{Z}_{t}^{\star}\right\}=\rho d t,
$$

and

$$
\Lambda(y)=\frac{\rho(\mu-r)}{f(y)}+\gamma(y) \sqrt{1-\rho^{2}}
$$

is a combined market price of risk. It describes the relationship between the physical measure under which the stock price is observed, and the risk-neutral measure under which the market prices derivative securities. See [2] for example. The price of the underlying stock is $S_{t}^{\varepsilon}$ and the volatility is a function $f$ of the process $Y_{t}^{\varepsilon}$. At the leading order $1 / \varepsilon$, that is omitting the $\Lambda$-term, $Y_{t}^{\varepsilon}$ is an Ornstein-Uhlenbeck $(\mathrm{OU})$ process which is fast mean-reverting with a normal invariant distribution $\mathcal{N}\left(m, \nu^{2}\right)$. Notice that in this framework the volatility driving process $\left(Y_{t}^{\varepsilon}\right)$ is autonomous in the sense that the coefficients in its defining SDE do not depend on the stock price $S_{t}^{\varepsilon}$.

In this fast mean-reverting stochastic volatility scenario, the volatility level fluctuates randomly around its mean level, and the epochs of high/low volatility are relatively short. This is the regime that we consider and under which we analyze the price of European derivatives. A derivative is defined by its nonnegative payoff function $H(S)$ which prescribes the value of the contract at its maturity time $T$ when the stock price is $S$. The payoff function must in general satisfy the integrability condition

$$
\mathbb{E}^{\star}\left\{H\left(S_{T}\right)^{2}\right\}<\infty,
$$

with $\mathbb{E}^{\star}$ denoting expectation with respect to $\mathbb{P}^{\star}$. Moreover, we assume:

1. The volatility is positive and bounded: there are constants $m_{1}$ and $m_{2}$ such that

$$
0<m_{1} \leq f(y) \leq m_{2}<\infty \quad \forall y \in \mathcal{R} .
$$

2. The volatility risk-premium is bounded:

$$
|\gamma(y)|<l<\infty \quad \forall y \in \mathcal{R} .
$$

for some constant $l$. 
It is convenient at this stage to make the change of variable

$$
X_{t}^{\varepsilon}=\log S_{t}^{\varepsilon}, \quad t \geq 0,
$$

and write the problem in terms of the processes $\left(X_{t}^{\varepsilon}, Y_{t}^{\varepsilon}\right)$ which satisfy, by Itô's formula the stochastic differential equations

$$
\begin{aligned}
& d X_{t}^{\varepsilon}=\left(r-\frac{1}{2} f\left(Y_{t}^{\varepsilon}\right)^{2}\right) d t+f\left(Y_{t}^{\varepsilon}\right) d W_{t}^{\star} \\
& d Y_{t}^{\varepsilon}=\left[\frac{1}{\varepsilon}\left(m-Y_{t}^{\varepsilon}\right)-\frac{\nu \sqrt{2}}{\sqrt{\varepsilon}} \Lambda\left(Y_{t}^{\varepsilon}\right)\right] d t+\frac{\nu \sqrt{2}}{\sqrt{\varepsilon}} d \hat{Z}_{t}^{\star}
\end{aligned}
$$

We also define the payoff function $h$ in terms of the log stock price via

$$
H\left(e^{x}\right)=h(x), \quad x \in \mathcal{R} .
$$

The price at time $t<T$ of this derivative is a function of the present value of the stock price, or equivalently the log stock price, $X_{t}^{\varepsilon}=x$ and the present value $Y_{t}^{\varepsilon}=y$ of the process driving the volatility. We denote this price by $P^{\varepsilon}(t, x, y)$. It is standard in finance to assume the price is given by (2.3) which is the expected discounted payoff under the risk-neutral probability measure $\mathbb{P}^{\star}$. See [1] for example.

$$
P^{\varepsilon}(t, x, y)=\mathbb{E}^{\star}\left\{e^{-r(T-t)} h\left(X_{T}^{\varepsilon}\right) \mid X_{t}^{\varepsilon}=x, Y_{t}^{\varepsilon}=y\right\}
$$

We shall also write these conditional expectations more compactly as

$$
P^{\varepsilon}(t, x, y)=\mathbb{E}^{\star} t, x, y\left\{e^{-r(T-t)} h\left(X_{T}^{\varepsilon}\right)\right\}
$$

Under the assumptions on the models considered and the payoff, $P^{\varepsilon}(t, x, y)$ is the unique classical solution to the associated backward Kolmogorov partial differential equation problem

$$
\begin{aligned}
\mathcal{L}^{\varepsilon} P^{\varepsilon} & =0, \\
P^{\varepsilon}(T, x, y) & =h(x)
\end{aligned}
$$

in $t<T, x, y \in \mathcal{R}$, where we have defined the operators

$$
\begin{aligned}
& \mathcal{L}^{\varepsilon}=\frac{1}{\varepsilon} \mathcal{L}_{0}+\frac{1}{\sqrt{\varepsilon}} \mathcal{L}_{1}+\mathcal{L}_{2} \\
& \mathcal{L}_{0}=\nu^{2} \frac{\partial^{2}}{\partial y^{2}}+(m-y) \frac{\partial}{\partial y}, \\
& \mathcal{L}_{1}=\sqrt{2} \rho \nu f(y) \frac{\partial^{2}}{\partial x \partial y}-\sqrt{2} \nu \Lambda(y) \frac{\partial}{\partial y}, \\
& \mathcal{L}_{2}=\frac{\partial}{\partial t}+\frac{1}{2} f(y)^{2} \frac{\partial^{2}}{\partial x^{2}}+\left(r-\frac{1}{2} f(y)^{2}\right) \frac{\partial}{\partial x}-r .
\end{aligned}
$$

The operator $\mathcal{L}_{0}$ is the infinitesimal generator of the OU process $\left(Y_{t}\right)$ defined by

$$
d Y_{t}=\left(m-Y_{t}\right) d t+\nu \sqrt{2} d \hat{Z}_{t}^{\star}
$$

$\mathcal{L}_{1}$ contains the mixed partial derivative due to the correlation and the derivative due to the market price of risk, and $\mathcal{L}_{2}$, also denoted by $\mathcal{L}_{B S}(f(y))$, is the Black-Scholes operator in the log variable and with volatility $f(y)$. 
3. Price approximation. We present here the formal asymptotic expansion computed as in $[2,3]$ which leads to a (first-order in $\sqrt{\varepsilon}$ ) approximation $P^{\varepsilon}(t, x, y) \approx Q^{\varepsilon}(t, x)$. In the next section we prove the convergence and accuracy as $\varepsilon \downarrow 0$ of this approximation which consists of the first two terms of the asymptotic price expansion:

$$
Q^{\varepsilon}(t, x)=P_{0}(t, x)+\sqrt{\varepsilon} P_{1}(t, x),
$$

which do not depend on $y$ and are derived as follows. We start by writing

$$
P^{\varepsilon}=Q^{\varepsilon}+\varepsilon Q_{2}+\varepsilon^{3 / 2} Q_{3}+\cdots=P_{0}+\sqrt{\varepsilon} P_{1}+\varepsilon Q_{2}+\varepsilon^{3 / 2} Q_{3}+\cdots,
$$

Substituting (3.1) into (2.4) leads to

$$
\begin{aligned}
& \frac{1}{\varepsilon} \mathcal{L}_{0} P_{0}+\frac{1}{\sqrt{\varepsilon}}\left(\mathcal{L}_{0} P_{1}+\mathcal{L}_{1} P_{0}\right) \\
& +\left(\mathcal{L}_{0} Q_{2}+\mathcal{L}_{1} P_{1}+\mathcal{L}_{2} P_{0}\right)+\sqrt{\varepsilon}\left(\mathcal{L}_{0} Q_{3}+\mathcal{L}_{1} Q_{2}+\mathcal{L}_{2} P_{1}\right)+\cdots=0
\end{aligned}
$$

We shall next obtain expressions for $P_{0}$ and $P_{1}$ by successively equating the four leading order terms in $(3.2)$ to zero. We let $\langle\cdot\rangle$ denote the averaging with respect to the invariant distribution $\mathcal{N}\left(m, \nu^{2}\right)$ of the OU process $Y$ introduced in $(2.8)$ :

$$
\langle g\rangle=\frac{1}{\nu \sqrt{2 \pi}} \int_{\mathcal{R}} g(y) e^{-(m-y)^{2} / 2 \nu^{2}} d y .
$$

Notice that this averaged quantity does not depend on $\varepsilon$.

Below, we will need to solve the Poisson equation associated with $\mathcal{L}_{0}$ :

$$
\mathcal{L}_{0} \chi+g=0
$$

which requires the solvability condition

$$
\langle g\rangle=0,
$$

in order to admit solutions with reasonable growth at infinity. Properties of this equation and its solutions are recalled in Appendix C.

Consider first the leading order term:

$$
\mathcal{L}_{0} P_{0}=0
$$

Since $\mathcal{L}_{0}$ takes derivatives with respect to $y$, any function independent of $y$ satisfies this equation. On the other hand $y$-dependent solutions exhibit the unreasonable growth $\exp \left(y^{2} / 2 \nu^{2}\right)$ at infinity. Therefore we seek solutions which are independent of $y: P_{0}=P_{0}(t, x)$ with the terminal condition $P_{0}(T, x)=h(x)$.

Consider next:

$$
\mathcal{L}_{0} P_{1}+\mathcal{L}_{1} P_{0}=0
$$

which corresponds to the second term in (3.2). Since $\mathcal{L}_{1}$ contains only terms with derivatives in $y$ it reduces to $\mathcal{L}_{0} P_{1}=0$ and, as for $P_{0}$, we seek again a function $P_{1}=P_{1}(t, x)$, independent of $y$, with a zero terminal condition $P_{1}(T, x)=0$. Hence, $Q^{\varepsilon}=P_{0}+\sqrt{\varepsilon} P_{1}$, the leading order approximation, does not depend on the current value of the volatility level.

The next equation

$$
\mathcal{L}_{0} Q_{2}+\mathcal{L}_{1} P_{1}+\mathcal{L}_{2} P_{0}=0,
$$

which corresponds to the third term in (3.2), reduces to the Poisson equation

$$
\mathcal{L}_{0} Q_{2}+\mathcal{L}_{2} P_{0}=0,
$$


since $\mathcal{L}_{1} P_{1}=0$. Its solvability condition

$$
\left\langle\mathcal{L}_{2} P_{0}\right\rangle=\left\langle\mathcal{L}_{2}\right\rangle P_{0}=0
$$

is the Black-Scholes PDE (in the log variable) with constant square volatility $\left\langle f^{2}\right\rangle$ :

$$
\left\langle\mathcal{L}_{2}\right\rangle P_{0}=\mathcal{L}_{B S}(\bar{\sigma}) P_{0}=\frac{\partial P_{0}}{\partial t}+\frac{1}{2} \bar{\sigma}^{2} \frac{\partial^{2} P_{0}}{\partial x^{2}}+\left(r-\frac{1}{2} \bar{\sigma}^{2}\right) \frac{\partial P_{0}}{\partial x}-r P_{0}=0,
$$

where we define the effective constant volatility $\bar{\sigma}$ by

$$
\bar{\sigma}^{2}=\left\langle f^{2}\right\rangle .
$$

We choose $P_{0}(t, x)$ to be the classical Black-Scholes price, solution of (3.7) with the terminal condition $P_{0}(T, x)=h(x)$.

Observe that $Q_{2}=-\mathcal{L}_{0}^{-1}\left(\mathcal{L}_{2}-\left\langle\mathcal{L}_{2}\right\rangle\right) P_{0}$ as a solution of the Poisson equation (3.6). This notation includes an additive constant in $y$ which will disappear when hit by the operator $\mathcal{L}_{1}$ as follows. The fourth term in (3.2) gives the equation :

$$
\mathcal{L}_{0} Q_{3}+\mathcal{L}_{1} Q_{2}+\mathcal{L}_{2} P_{1}=0
$$

This is a Poisson equation in $Q_{3}$, and its solvability condition gives

$$
\left\langle\mathcal{L}_{2}\right\rangle P_{1}=-\left\langle\mathcal{L}_{1} Q_{2}\right\rangle=\left\langle\mathcal{L}_{1} \mathcal{L}_{0}^{-1}\left(\mathcal{L}_{2}-\left\langle\mathcal{L}_{2}\right\rangle\right)\right\rangle P_{0},
$$

which, with its zero terminal condition, determines $P_{1}$ as a solution of a Black-Scholes equation with constant square volatility $\left\langle f^{2}\right\rangle$ and a source term. Using the expressions for $\mathcal{L}_{i}$ one can rewrite the source as:

$$
\begin{aligned}
\left\langle\mathcal{L}_{1} \mathcal{L}_{0}^{-1}\left(\mathcal{L}_{2}-\left\langle\mathcal{L}_{2}\right\rangle\right) P_{0}\right. & =\left\langle\mathcal{L}_{1} \mathcal{L}_{0}^{-1}\left(f(y)^{2}-\left\langle f^{2}\right\rangle\right)\right\rangle \frac{1}{2}\left(\frac{\partial^{2}}{\partial x^{2}}-\frac{\partial}{\partial x}\right) P_{0} \\
& =\left(v_{3} \frac{\partial^{3}}{\partial x^{3}}+\left(v_{2}-3 v_{3}\right) \frac{\partial^{2}}{\partial x^{2}}+\left(2 v_{3}-v_{2}\right) \frac{\partial}{\partial x}\right) P_{0},
\end{aligned}
$$

where

$$
\begin{aligned}
& v_{2}=\frac{\nu}{\sqrt{2}}\left(2 \rho\left\langle f \phi^{\prime}\right\rangle-\left\langle\Lambda \phi^{\prime}\right\rangle\right) \\
& v_{3}=\frac{\rho \nu}{\sqrt{2}}\left\langle f \phi^{\prime}\right\rangle,
\end{aligned}
$$

and $\phi$ is a solution of the Poisson equation:

$$
\mathcal{L}_{0} \phi(y)=f(y)^{2}-\left\langle f^{2}\right\rangle .
$$

We can therefore conclude:

1. The first term $P_{0}$ is chosen to be the solution of the "homogenized" PDE problem (3.7). In other words, $P_{0}$ is simply the Black-Scholes price of the derivative computed with the effective volatility $\bar{\sigma}$.

2. The second term, or correction to the Black-Scholes price, is given explicitly, as a linear combination of the first three derivatives of $P_{0}$, by

$$
\sqrt{\varepsilon} P_{1}=-(T-t)\left(V_{3}^{\varepsilon} \frac{\partial^{3}}{\partial x^{3}}+\left(V_{2}^{\varepsilon}-3 V_{3}^{\varepsilon}\right) \frac{\partial^{2}}{\partial x^{2}}+\left(2 V_{3}^{\varepsilon}-V_{2}^{\varepsilon}\right) \frac{\partial}{\partial x}\right) P_{0},
$$

with

$$
V_{2,3}^{\varepsilon}=\sqrt{\varepsilon} v_{2,3},
$$

since it is easily seen, by using $\left\langle\mathcal{L}_{2}\right\rangle P_{0}=0$, that equation (3.9) is satisfied, and that, on the other hand, the terminal condition $P_{1}(T, x)=0$ is satisfied when $\lim _{t \rightarrow T}(T-t) \frac{\partial^{i} P_{0}}{\partial x^{i}}=0$ for $i=1,2,3$. 
Essential instruments in financial markets are put and call options for which the payoff function $H(S)$ is piecewise linear. We shall focus on call options:

$$
H(S)=(S-K)^{+} \quad \Rightarrow \quad h(x)=\left(e^{x}-K\right)^{+}
$$

for some given strike price $K>0$. Notice that $h$ is only $\mathcal{C}^{0}$ smooth with a discontinuous first derivative at the $\operatorname{kink} x=\log K$, (at the money in financial terms). Nonetheless, at $t<T$, the Black-Scholes pricing function $P_{0}(t, x)$ is smooth and $P_{1}(t, x)$ is well-defined, but second and higher derivatives of $P_{0}$ with respect to $x$ blow up as $t \rightarrow T$ (at the money).

Our main result on the accuracy of the approximation $Q^{\varepsilon}=P_{0}+\sqrt{\varepsilon} P_{1}$ is as follows:

THEOREM 3.1. Under the assumptions (1) and (2) above, at a fixed point $t<T, x, y \in \mathcal{R}$, the accuracy of the approximation of call prices is given by

$$
\lim _{\varepsilon \downarrow 0} \frac{\left|P^{\varepsilon}(t, x, y)-Q^{\varepsilon}(t, x)\right|}{\varepsilon|\log \varepsilon|^{1+p}}=0,
$$

for any $p>0$. Observe that this pointwise approximation is the sense of accuracy needed in finance applications since option prices are computed at given values of $(t, x, y)$.

Before giving in the next Section the proof of Theorem 3.1, we comment on the interpretation of the approximation and on the validity of the result for more general payoffs.

\section{Financial interpretation of the approximation.}

In order to give a meaningful interpretation to the leading order term and the correction in our price approximation it is convenient to return to the variable $S$, the underlying price. With a slight abuse of notation we denote the call option price approximation by $P_{0}(t, S)+\sqrt{\varepsilon} P_{1}(t, S)$. Indeed the leading order term $P_{0}(t, S)$ is the standard Black-Scholes price of the call option computed at the effective constant volatility $\bar{\sigma}$. From (3.12), one can easily deduce that

$$
\sqrt{\varepsilon} P_{1}(t, S)=-(T-t)\left(V_{2}^{\varepsilon} S^{2} \frac{\partial^{2} P_{0}}{\partial S^{2}}+V_{3}^{\varepsilon} S^{3} \frac{\partial^{3} P_{0}}{\partial S^{3}}\right),
$$

which shows that the correction is a combination of the two greeks Gamma and Epsilon, as introduced in [2]. This correction can alternatively be written in the form

$$
\sqrt{\varepsilon} P_{1}(t, S)=-(T-t)\left(\left(V_{2}^{\varepsilon}-2 V_{3}^{\varepsilon}\right) S^{2} \frac{\partial^{2} P_{0}}{\partial S^{2}}+V_{3}^{\varepsilon} S \frac{\partial}{\partial S}\left(S^{2} \frac{\partial^{2} P_{0}}{\partial S^{2}}\right)\right) .
$$

Using the classical relation between Gamma and Vega for Black-Scholes prices of European derivatives

$$
\frac{\partial P_{0}}{\partial \sigma}=(T-t) \sigma S^{2} \frac{\partial^{2} P_{0}}{\partial S^{2}}
$$

which is easily obtained by differentiating the Black-Scholes PDE with respect to $\sigma$, one can rewrite the correction as:

$$
\sqrt{\varepsilon} P_{1}(t, S)=-\frac{1}{\bar{\sigma}}\left(\left(V_{2}^{\varepsilon}-2 V_{3}^{\varepsilon}\right) \frac{\partial P_{0}}{\partial \sigma}+V_{3}^{\varepsilon} S \frac{\partial}{\partial S}\left(\frac{\partial P_{0}}{\partial \sigma}\right)\right) .
$$

Therefore the price correction is a combination of the Vega and the Delta-Vega of the Black-Scholes price. The Vega term corresponds simply to a volatility level correction. The Delta-Vega term is proportional to the correlation coefficient $\rho$ and captures the main effect of skewness in implied volatility as discussed in detail in [2].

\section{Other payoff functions.}

The main idea of the proof presented in the next Section is a regularization of the payoff which does not rely on the particular choice of a call option. The only place where we use the explicit 
Black-Scholes formula for a call option is in the computation (B.1) of the successive derivatives $\partial_{x}^{n} P_{0}^{\delta}$ carried out in the Appendix B. Note that if we had started with a payoff function $h$ which is continuous and piecewise smooth (a call option being a particular case), then $P_{0}^{\delta}$, the solution of the parabolic PDE (3.7), is an integral of the payoff function with respect to a normal density as in the case of a call option. The first derivative with respect to $x$ can be taken on the payoff function and the higher order derivatives can then be taken on the normal density as detailed in Appendix B for a call option. Therefore Theorem 3.1 remains valid for general European claims with continuous payoffs that have singular behavior in their derivatives.

Numerical illustration. To illustrate the asymptotic approximation, we compare the approximation

$$
Q^{\varepsilon}=P_{0}+\sqrt{\varepsilon} P_{1}
$$

with a numerical solution of the PDE (2.4) for a particular stochastic volatility model and a call option with strike price $K=100$ and three months from expiration. (In practice, the asymptotic approximation is not used in this manner because of the difficulties of estimating the volatility parameters precisely; instead the parameters of the approximation $V_{2}^{\varepsilon}$ and $V_{3}^{\varepsilon}$ are estimated directly from observed options prices, as described in [2]).

We choose $f(y)=e^{y}$, where this is understood to stand for a cutoff version of the exponential function with the cutoffs (above and below) sufficiently large and small respectively so as not to affect the calculations within the accuracy of our comparisons. We use the parameter values

$$
\begin{aligned}
\varepsilon=1 / 200, \quad m & =\log 0.1, \quad \nu=1 / \sqrt{2}, \quad \rho=-0.2, \\
\mu & =0.2, \quad r=0.04,
\end{aligned}
$$

and choose the volatility risk premium $\gamma \equiv 0$. It follows from explicit calculations that the parameters for the asymptotic approximation are

$$
\bar{\sigma}=0.165, \quad V_{2}^{\varepsilon}=-3.30 \times 10^{-4}, \quad V_{3}^{\varepsilon}=8.48 \times 10^{-5} .
$$

Figure 3.1 shows the numerical solution from an implicit finite-difference approximation at two levels of the current volatility $e^{y}$, one at the long-run mean-level $\bar{\sigma}$, and one far above it $(0.607)$. These are compared to the asymptotic approximation which does not depend on the current volatility level. In the range $0.95 \leq K / S \leq 1.04$ shown in the picture on the right, the maximum deviation of the asymptotic approximation from the price with the higher volatility is by $9 \%$ of the latter price, and the maximum deviation of the asymptotic approximation from the price with the lower volatility is by $2.1 \%$ of this price.

4. Derivation of the accuracy of the price approximation. In order to prove Theorem 3.1 , we introduce in the next section the regularized price, $P^{\varepsilon, \delta}$, the price of a slightly smoothed call option, with $\delta$ being the (small) smoothing parameter. We denote the associated price approximation $Q^{\varepsilon, \delta}$. The proof then involves showing that (i) $P^{\varepsilon} \approx P^{\varepsilon, \delta}$, (ii) $Q^{\varepsilon, \delta} \approx Q^{\varepsilon}$, (iii) $P^{\varepsilon, \delta} \approx Q^{\varepsilon, \delta}$, and controlling the accuracy in these approximations by choosing $\delta$ appropriately.

4.1. Regularization. We begin by regularizing the payoff, which is a call option, by replacing it with the Black-Scholes price of a call with volatility $\bar{\sigma}$ and time to maturity $\delta$. We define

$$
h^{\delta}(x):=C_{B S}(T-\delta, x ; K, T ; \bar{\sigma}),
$$

where $C_{B S}(t, x ; K, T ; \bar{\sigma})$ denotes the Black-Scholes call option price as a function of current time $t$, $\log$ stock price $x$, strike price $K$, expiration date $T$ and volatility $\bar{\sigma}$. It is given by

$$
\begin{aligned}
C_{B S}(t, x ; K, T ; \bar{\sigma}) & =P_{0}(t, x ; K, T ; \bar{\sigma})=e^{x} N\left(d_{1}\right)-K e^{-r \frac{\tau^{2}}{\bar{\sigma}}} N\left(d_{2}\right) \\
N(x) & =\frac{1}{\sqrt{2 \pi}} \int_{-\infty}^{x} e^{-y^{2} / 2} d y \\
d_{1} & =\frac{x-\log K}{\tau}+b \tau \\
d_{2} & =d_{1}-\tau,
\end{aligned}
$$



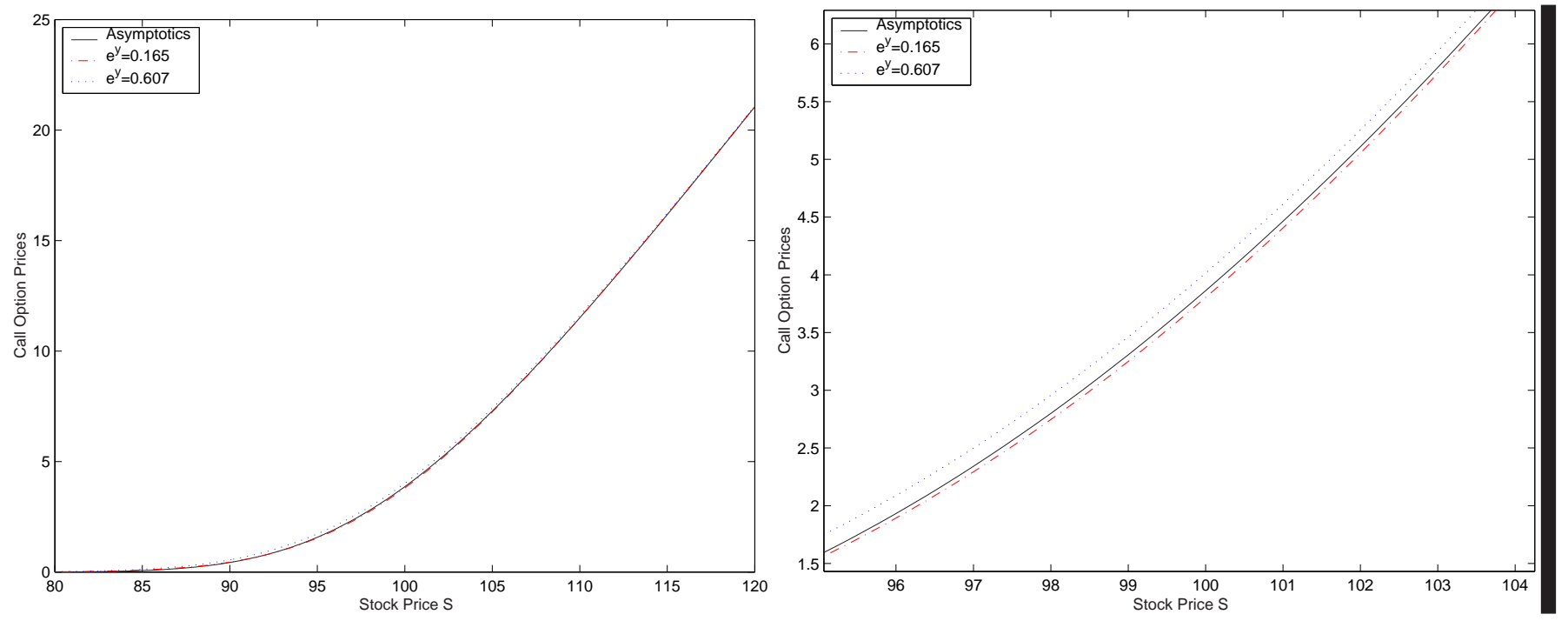

FIG. 3.1. Call option prices 3 months from maturity as a function of the current stock price $S$. The strike price is $K=100$ and the picture on the right focuses on the region "around the money".

where we define

$$
\tau=\bar{\sigma} \sqrt{T-t} \quad b=\frac{r}{\bar{\sigma}^{2}}+\frac{1}{2}
$$

For $\delta>0$, this new payoff is $\mathcal{C}^{\infty}$. The price $P^{\varepsilon, \delta}(t, x, y)$ of the option with the regularized payoff solves

$$
\begin{aligned}
\mathcal{L}^{\varepsilon} P^{\varepsilon, \delta} & =0 \\
P^{\varepsilon, \delta}(T, x, y) & =h^{\delta}(x) .
\end{aligned}
$$

4.2. Main convergence result. Let $Q^{\varepsilon, \delta}(t, x)$ denote the first-order approximation to the regularized option price:

$$
P^{\varepsilon, \delta} \approx Q^{\varepsilon, \delta} \equiv P_{0}^{\delta}+\sqrt{\varepsilon} P_{1}^{\delta},
$$

where

$$
\begin{aligned}
P_{0}^{\delta}(t, x) & =C_{B S}(t-\delta, x ; K, T ; \bar{\sigma}) \\
\sqrt{\varepsilon} P_{1}^{\delta} & =-(T-t)\left(V_{3}^{\varepsilon} \frac{\partial^{3}}{\partial x^{3}}+\left(V_{2}^{\varepsilon}-3 V_{3}^{\varepsilon}\right) \frac{\partial^{2}}{\partial x^{2}}+\left(2 V_{3}^{\varepsilon}-V_{2}^{\varepsilon}\right) \frac{\partial}{\partial x}\right) P_{0}^{\delta}
\end{aligned}
$$

We establish the following pathway to proving Theorem 3.1 where constants may depend on $(t, T, x, y)$ but not on $(\varepsilon, \delta)$ :

Lemma 4.1. Fix the point $(t, x, y)$ where $t<T$. There exist constants $\bar{\delta}_{1}>0, \bar{\varepsilon}_{1}>0$ and $c_{1}>0$ such that

$$
\left|P^{\varepsilon}(t, x, y)-P^{\varepsilon, \delta}(t, x, y)\right| \leq c_{1} \delta
$$

for all $0<\delta<\bar{\delta}_{1}$ and $0<\varepsilon<\bar{\varepsilon}_{1}$. This establishes that the solutions to the regularized and unregularized problems are close.

Lemma 4.2. Fix the point $(t, x, y)$ where $t<T$. There exist constants $\bar{\delta}_{2}>0, \bar{\varepsilon}_{2}>0$ and $c_{2}>0$ such that

$$
\left|Q^{\varepsilon}(t, x)-Q^{\varepsilon, \delta}(t, x)\right| \leq c_{2} \delta
$$


for all $0<\delta<\bar{\delta}_{2}$ and $0<\varepsilon<\bar{\varepsilon}_{2}$. This establishes that the first-order asymptotic approximations to the regularized and unregularized problems are close.

Lemma 4.3. Fix the point $(t, x, y)$ where $t<T$. There exist constants $\bar{\delta}_{3}>0, \bar{\varepsilon}_{3}>0$ and $c_{3}>0$ such that

$$
\left|P^{\varepsilon, \delta}(t, x, y)-Q^{\varepsilon, \delta}(t, x)\right| \leq c_{3}\left(\varepsilon|\log \delta|+\varepsilon \sqrt{\frac{\varepsilon}{\delta}}+\varepsilon\right)
$$

for all $0<\delta<\bar{\delta}_{3}$ and $0<\varepsilon<\bar{\varepsilon}_{3}$. This establishes that for fixed $\delta$, the approximation to the regularized problem converges to the regularized price as $\varepsilon \downarrow 0$.

The convergence result follows from these Lemmas:

Proof of Theorem 3.1. Take $\bar{\delta}=\min \left(\bar{\delta}_{1}, \bar{\delta}_{2}, \bar{\delta}_{3}\right)$ and $\bar{\varepsilon}=\min \left(\bar{\varepsilon}_{1}, \bar{\varepsilon}_{2}, \bar{\varepsilon}_{3}\right)$. Then using Lemmas 4.1, 4.2 and 4.3 , we obtain

$$
\begin{aligned}
\left|P^{\varepsilon}-Q^{\varepsilon}\right| & \leq\left|P^{\varepsilon}-P^{\varepsilon, \delta}\right|+\left|P^{\varepsilon, \delta}-Q^{\varepsilon, \delta}\right|+\left|Q^{\varepsilon, \delta}-Q^{\varepsilon}\right| \\
& \leq 2 \max \left(c_{1}, c_{2}\right) \delta+c_{3}\left(\varepsilon|\log \delta|+\varepsilon \sqrt{\frac{\varepsilon}{\delta}}+\varepsilon\right),
\end{aligned}
$$

for $0<\delta<\bar{\delta}$ and $0<\varepsilon<\bar{\varepsilon}$, where the functions are evaluated at the fixed $(t, x, y)$. Taking $\delta=\varepsilon$, we have

$$
\left|P^{\varepsilon}-Q^{\varepsilon}\right| \leq c_{5}(\varepsilon+\varepsilon|\log \varepsilon|),
$$

for some fixed $c_{5}>0$, and Theorem 3.1 follows.

A general conclusion to our work is given in Section 6 after the proofs of Lemmas 4.1,4.2 and 4.3 given in the following section.

\section{Proof of Lemmas.}

5.1. Proof of Lemma 4.1. We use the probabilistic representation of the price given as the expected discounted payoff with respect to the risk-neutral pricing equivalent martingale measure $\mathbb{P}^{\star}$.

$$
P^{\varepsilon, \delta}(t, x, y)=\mathbb{E}_{t, x, y}^{\star}\left\{e^{-r(T-t)} h^{\delta}\left(X_{T}^{\varepsilon}\right)\right\} .
$$

We define the new process $\left(\tilde{X}_{t}^{\varepsilon}\right)$ by

$$
d \tilde{X}_{t}^{\varepsilon}=\left(r-\frac{1}{2} \tilde{f}\left(t, Y_{t}^{\varepsilon}\right)^{2}\right) d t+\tilde{f}\left(t, Y_{t}^{\varepsilon}\right)\left(\sqrt{1-\rho^{2}} d \hat{W}_{t}^{\star}+\rho d \hat{Z}_{t}^{\star}\right)
$$

where $\left(\hat{W}_{t}^{\star}\right)$ is a Brownian motion independent of $\left(\hat{Z}_{t}^{\star}\right),\left(Y_{t}^{\varepsilon}\right)$ is still a solution of $(2.2)$ and

$$
\tilde{f}(t, y)= \begin{cases}f(y) & \text { for } t \leq T \\ \bar{\sigma} & \text { for } t>T\end{cases}
$$

Then we can write

$$
P^{\varepsilon, \delta}(t, x, y)=\mathbb{E}^{\star} t, x, y\left\{e^{-r(T-t+\delta)} h\left(\tilde{X}_{T+\delta}^{\varepsilon}\right)\right\}
$$

and

$$
P^{\varepsilon}(t, x, y)=\mathbb{E}^{\star}{ }_{t, x, y}\left\{e^{-r(T-t)} h\left(\tilde{X}_{T}^{\varepsilon}\right)\right\} .
$$

Next we use the iterated expectations formula

$$
\begin{aligned}
& P^{\varepsilon, \delta}(t, x, y)-P^{\varepsilon}(t, x, y)= \\
& \mathbb{E}^{\star}{ }_{t, x, y}\left\{\mathbb{E}^{\star}\left\{e^{-r(T-t+\delta)} h\left(\tilde{X}_{T+\delta}^{\varepsilon}\right)-e^{-r(T-t)} h\left(\tilde{X}_{T}^{\varepsilon}\right) \mid\left(\hat{Z}_{s}^{\star}\right)_{t \leq s \leq T}\right\}\right\},
\end{aligned}
$$


to obtain a representation of this price difference in terms of the Black-Scholes function $P_{0}$ which is smooth away from the terminal date $T$. In the uncorrelated case it corresponds to the Hull-White formula [7]. In the correlated case, as considered here, this formula is in [8], and can be found in $[2](2.8 .3)$. It is simple to compute explicitly the conditional distribution $\mathcal{D}\left(\tilde{X}_{T}^{\varepsilon} \mid\left(\hat{Z}_{s}^{\star}\right)_{t \leq s \leq T}, \tilde{X}_{t}^{\varepsilon}\right)$ of $\tilde{X}_{T}^{\varepsilon}$ given the path of the second Brownian motion $\left(\hat{Z}_{s}^{\star}\right)_{t \leq s \leq T}$. One obtains

$$
\mathcal{D}\left(\tilde{X}_{T}^{\varepsilon} \mid\left(\hat{Z}_{s}^{\star}\right)_{t \leq s \leq T}, \tilde{X}_{t}^{\varepsilon}=x\right)=\mathcal{N}\left(m_{1}^{\varepsilon}, v_{1}^{\varepsilon}\right),
$$

where the mean and variance are given by

$$
\begin{aligned}
m_{1}^{\varepsilon} & =x+\xi_{t, T}+\left(r-\frac{1}{2} \bar{\sigma}_{\rho}^{2}\right)(T-t) \\
v_{1}^{\varepsilon} & =\bar{\sigma}_{\rho}^{2}(T-t)
\end{aligned}
$$

and we define

$$
\begin{aligned}
\xi_{t, T} & =\rho \int_{t}^{T} \tilde{f}\left(s, Y_{s}^{\varepsilon}\right) d \hat{Z}_{s}^{\star}-\frac{1}{2} \rho^{2} \int_{t}^{T} \tilde{f}\left(s, Y_{s}^{\varepsilon}\right)^{2} d s \\
\bar{\sigma}_{\rho}^{2} & =\frac{1-\rho^{2}}{T-t} \int_{t}^{T} \tilde{f}\left(s, Y_{s}^{\varepsilon}\right)^{2} d s .
\end{aligned}
$$

It follows from the calculation that leads to the Black-Scholes formula that

$$
\mathbb{E}^{\star}{ }_{t, x, y}\left\{e^{-r(T-t)} h\left(\tilde{X}_{T}^{\varepsilon}\right) \mid\left(\hat{Z}_{s}^{\star}\right)_{t \leq s \leq T}\right\}=P_{0}\left(t, \tilde{X}_{t}^{\varepsilon}+\xi_{t, T} ; K, T ; \bar{\sigma}_{\rho}\right) .
$$

Similarly, we compute

$$
\mathcal{D}\left(\tilde{X}_{T+\delta}^{\varepsilon} \mid\left(\hat{Z}_{s}^{\star}\right)_{t \leq s \leq T}, \tilde{X}_{t}^{\varepsilon}=x\right)=\mathcal{N}\left(m_{2}^{\varepsilon}, v_{2}^{\varepsilon}\right)
$$

where the mean and variance are given by

$$
\begin{aligned}
m_{2}^{\varepsilon} & =x+\xi_{t, T}+r \delta+\left(r-\frac{1}{2} \tilde{\sigma}_{\rho, \delta}^{2}\right)(T-t) \\
v_{2}^{\varepsilon} & =\tilde{\sigma}_{\rho, \delta}^{2}(T-t)
\end{aligned}
$$

and we define

$$
\tilde{\sigma}_{\rho, \delta}^{2}=\bar{\sigma}_{\rho}^{2}+\frac{\delta \bar{\sigma}^{2}}{T-t} .
$$

Therefore

$$
\mathbb{E}_{t, x, y}^{\star}\left\{e^{-r(T-t+\delta)} h\left(\tilde{X}_{T+\delta}^{\varepsilon}\right) \mid\left(\hat{Z}_{s}^{\star}\right)_{t \leq s \leq T}\right\}=P_{0}\left(t, \tilde{X}_{t}^{\varepsilon}+\xi_{t, T}+r \delta ; K, T ; \tilde{\sigma}_{\rho, \delta}\right),
$$

and we can write

$$
\begin{aligned}
& P^{\varepsilon, \delta}(t, x, y)-P^{\varepsilon}(t, x, y)= \\
& \mathbb{E}^{\star}{ }_{t, x, y}\left\{P_{0}\left(t, x+\xi_{t, T}+r \delta ; K, T ; \tilde{\sigma}_{\rho, \delta}\right)-P_{0}\left(t, x+\xi_{t, T} ; K, T ; \bar{\sigma}_{\rho}\right)\right\} .
\end{aligned}
$$

Using the explicit representation (4.1) and that $\bar{\sigma}_{\rho}$ is bounded above and below as $f(y)$ is, we find

$$
\left|P_{0}\left(t, x+\xi_{t, T}+r \delta ; K, T ; \tilde{\sigma}_{\rho, \delta}\right)-P_{0}\left(t, x+\xi_{t, T} ; K, T ; \bar{\sigma}_{\rho}\right)\right| \leq \delta c_{1}\left(e^{\xi_{t, T}}\left[\left|\xi_{t, T}\right|+1\right]+1\right)
$$

for some $c_{1}$ and for $\delta$ small enough. Using the definition (5.1) of $\xi_{t, T}$ and the existence of its exponential moments, we thus find that

$$
\left|P^{\varepsilon}(t, x, y)-P^{\varepsilon, \delta}(t, x, y)\right| \leq c_{2} \delta
$$

for some $c_{2}$ and for $\delta$ small enough. 
5.2. Proof of Lemma 4.2. From the definition (3.12) of the correction $\sqrt{\varepsilon} P_{1}$ and the corresponding definition (4.3) of the correction $\sqrt{\varepsilon} P_{1}^{\delta}$ we deduce

$$
\begin{aligned}
& Q^{\varepsilon, \delta}-Q^{\varepsilon} \\
& =\left(1-(T-t)\left(V_{3}^{\varepsilon} \frac{\partial^{3}}{\partial x^{3}}+\left(V_{2}^{\varepsilon}-3 V_{3}^{\varepsilon}\right) \frac{\partial^{2}}{\partial x^{2}}+\left(2 V_{3}^{\varepsilon}-V_{2}^{\varepsilon}\right) \frac{\partial}{\partial x}\right)\right)\left(P_{0}^{\delta}-P_{0}\right) .
\end{aligned}
$$

From the definition (3.10) of the $v_{i}$ 's, the definition (3.13) of the $V_{i}$ 's and the bounds on the solution of the Poisson equation (3.11) given in Appendix C, it follows that

$$
\max \left(\left|V_{2}^{\varepsilon}\right|,\left|V_{3}^{\varepsilon}\right|\right) \leq c_{1} \sqrt{\varepsilon}
$$

for some constant $c_{1}>0$. Notice that we can write

$$
P_{0}^{\delta}(t, x)=P_{0}(t-\delta, x) .
$$

Using the explicit formula (4.1), it is easily seen that $P_{0}$ and its successive derivatives with respect to $x$ are differentiable in $t$ at any $t<T$. Therefore we conclude that for $(t, x, y)$ fixed with $t<T$ :

$$
\left|Q^{\varepsilon}(t, x)-Q^{\varepsilon, \delta}(t, x)\right| \leq c_{2} \delta
$$

for some $c_{2}>0$ and $\delta$ small enough.

5.3. Proof of Lemma 4.3. We first introduce some additional notation. Define the error $Z^{\varepsilon, \delta}$ in the approximation for the regularized problem by

$$
P^{\varepsilon, \delta}=P_{0}^{\delta}+\sqrt{\varepsilon} P_{1}^{\delta}+\varepsilon Q_{2}^{\delta}+\varepsilon^{3 / 2} Q_{3}^{\delta}-Z^{\varepsilon, \delta},
$$

for $Q_{2}^{\delta}$ and $Q_{3}^{\delta}$ stated below in (5.3) and (5.4). Setting

$$
\mathcal{L}^{\varepsilon}=\frac{1}{\varepsilon} \mathcal{L}_{0}+\frac{1}{\sqrt{\varepsilon}} \mathcal{L}_{1}+\mathcal{L}_{2}
$$

one can write

$$
\begin{aligned}
\mathcal{L}^{\varepsilon} Z^{\varepsilon, \delta}= & \mathcal{L}^{\varepsilon}\left(P_{0}^{\delta}+\sqrt{\varepsilon} P_{1}^{\delta}+\varepsilon Q_{2}^{\delta}+\varepsilon^{3 / 2} Q_{3}^{\delta}-P^{\varepsilon, \delta}\right) \\
= & \frac{1}{\varepsilon} \mathcal{L}_{0} P_{0}^{\delta}+\frac{1}{\sqrt{\varepsilon}}\left(\mathcal{L}_{0} P_{1}^{\delta}+\mathcal{L}_{1} P_{0}^{\delta}\right) \\
& +\left(\mathcal{L}_{0} Q_{2}^{\delta}+\mathcal{L}_{1} P_{1}^{\delta}+\mathcal{L}_{2} P_{0}^{\delta}\right)+\sqrt{\varepsilon}\left(\mathcal{L}_{0} Q_{3}^{\delta}+\mathcal{L}_{1} Q_{2}^{\delta}+\mathcal{L}_{2} P_{1}^{\delta}\right) \\
& +\varepsilon\left(\mathcal{L}_{1} Q_{3}^{\delta}+\mathcal{L}_{2} Q_{2}^{\delta}+\sqrt{\varepsilon} \mathcal{L}_{2} Q_{3}^{\delta}\right) \\
= & \varepsilon\left(\mathcal{L}_{1} Q_{3}^{\delta}+\mathcal{L}_{2} Q_{2}^{\delta}\right)+\varepsilon^{3 / 2} \mathcal{L}_{2} Q_{3}^{\delta} \equiv G^{\varepsilon, \delta}
\end{aligned}
$$

because $P^{\varepsilon, \delta}$ solves the original equation $\mathcal{L}^{\varepsilon} P^{\varepsilon, \delta}=0$ and we choose $P_{0}^{\delta}, P_{1}^{\delta}, Q_{2}^{\delta}$ and $Q_{3}^{\delta}$ to cancel the first four terms. In particular, we choose

$$
Q_{2}^{\delta}(t, x, y)=-\frac{1}{2} \phi(y)\left(\frac{\partial^{2} P_{0}^{\delta}}{\partial x^{2}}-\frac{\partial P_{0}^{\delta}}{\partial x}\right)
$$

so that

$$
\mathcal{L}_{0} Q_{2}^{\delta}=-\mathcal{L}_{2} P_{0}^{\delta}
$$

(with an "integration constant" arbitrarily set to zero) whereas $Q_{3}^{\delta}$ is a solution of the Poisson equation

$$
\mathcal{L}_{0} Q_{3}^{\delta}=-\left(\mathcal{L}_{1} Q_{2}^{\delta}+\mathcal{L}_{2} P_{1}^{\delta}\right),
$$


where the centering condition is ensured by our choice of $P_{1}^{\delta}$.

At the terminal time $T$ we have

$$
Z^{\varepsilon, \delta}(T, x, y)=\varepsilon\left(Q_{2}^{\delta}(T, x, y)+\sqrt{\varepsilon} Q_{3}^{\delta}(T, x, y)\right) \equiv H^{\varepsilon, \delta}(x, y),
$$

where we have used the terminal conditions $P^{\varepsilon, \delta}(T, x, y)=P_{0}^{\delta}(T, x)=h^{\delta}(x)$ and $P_{1}^{\delta}(T, x)=0$. This assumes smooth derivatives of $P_{0}^{\delta}$ in the domain $t \leq T$ which is the case because $h^{\delta}$ is smooth. It is shown in Appendix A that the source term $G^{\varepsilon, \delta}(t, x, y)$ on the right-side of equation (5.2) can be written in the form

$$
\begin{aligned}
G^{\varepsilon, \delta}= & \varepsilon\left(\sum_{i=1}^{4} g_{i}^{(1)}(y) \frac{\partial^{i}}{\partial x^{i}} P_{0}^{\delta}+(T-t) \sum_{i=1}^{6} g_{i}^{(2)}(y) \frac{\partial^{i}}{\partial x^{i}} P_{0}^{\delta}\right) \\
& +\varepsilon^{3 / 2}\left(\sum_{i=1}^{5} g_{i}^{(3)}(y) \frac{\partial^{i}}{\partial x^{i}} P_{0}^{\delta}+(T-t) \sum_{i=1}^{7} g_{i}^{(4)}(y) \frac{\partial^{i}}{\partial x^{i}} P_{0}^{\delta}\right) .
\end{aligned}
$$

In Appendix A we also show that the terminal condition $H^{\varepsilon, \delta}(x, y)$ in (5.5) can be written

$$
H^{\varepsilon, \delta}(x, y)=\varepsilon\left(\sum_{i=1}^{2} h_{i}^{(1)}(y) \frac{\partial^{i}}{\partial x^{i}} P_{0}^{\delta}(T, x)\right)+\varepsilon^{3 / 2}\left(\sum_{i=1}^{3} h_{i}^{(2)}(y) \frac{\partial^{i}}{\partial x^{i}} P_{0}^{\delta}(T, x)\right) .
$$

To bound the contributions from the source term and terminal conditions we need the following two Lemmas that are derived in Appendix $\mathrm{C}$ and Appendix B respectively:

LEMmA 5.1. Let $\chi=g_{i}^{(j)}$ or $\chi=h_{i}^{(j)}$ with the functions $g_{i}^{(j)}$ and $h_{i}^{(j)}$ being defined in (5.6) and (5.7). Then there exists a constant $c>0$ (which may depend on $y$ ) such that $\mathbb{E}^{\star}\left\{\left|\chi\left(Y_{s}^{\varepsilon}\right)\right| Y_{t}^{\varepsilon}=y\right\} \leq$ $c<\infty$ for $t \leq s \leq T$.

Lemma 5.2. Assume $T-t>\Delta>0$ and $\mathbb{E}^{\star}\left\{\left|\chi\left(Y_{s}^{\varepsilon}\right)\right| Y_{t}^{\varepsilon}=y\right\} \leq c_{1}<\infty$ for some constant $c_{1}$ then there exist constants $c_{2}>0$ and $\bar{\delta}>0$ such that for $\delta<\bar{\delta}$ and $t \leq s \leq T$

$$
\left|\mathbb{E}^{\star} t, x, y\left\{\sum_{i=1}^{n} \chi\left(Y_{s}^{\varepsilon}\right) \frac{\partial^{i}}{\partial x^{i}} P_{0}^{\delta}\left(s, X_{s}^{\varepsilon}\right)\right\}\right| \leq c_{2}[T+\delta-s]^{\min [0,1-n / 2]},
$$

and consequently

$$
\begin{aligned}
& \left|\mathbb{E}^{\star}{ }_{t, x, y}\left\{\int_{t}^{T}(T-s)^{p} \sum_{i=1}^{n} e^{-r(s-t)} \chi\left(Y_{s}^{\varepsilon}\right) \frac{\partial^{i}}{\partial x^{i}} P_{0}^{\delta}\left(s, X_{s}^{\varepsilon}\right) d s\right\}\right| \\
& \leq \begin{cases}c_{2}|\log (\delta)| & \text { for } n=4+2 p \\
c_{2} \delta^{\min [0, p+(4-n) / 2]} & \text { else } .\end{cases}
\end{aligned}
$$

Proof of Lemma 4.3 $H^{\varepsilon, \delta}$ :

We use the probabilistic representation of equation (5.2), $\mathcal{L}^{\varepsilon} Z^{\varepsilon, \delta}=G^{\varepsilon, \delta}$ with terminal condition

$$
Z^{\varepsilon, \delta}(t, x, y)=\mathbb{E}_{t, x, y}^{\star}\left\{e^{-r(T-t)} H^{\varepsilon, \delta}\left(X_{T}^{\varepsilon}, Y_{T}^{\varepsilon}\right)-\int_{t}^{T} e^{-r(s-t)} G^{\varepsilon, \delta}\left(s, X_{s}^{\varepsilon}, Y_{s}^{\varepsilon}\right) d s\right\} .
$$

From Lemma 5.2 it follows that there exists a constant $c>0$ such that

$$
\begin{gathered}
\left|\mathbb{E}_{t, x, y}^{\star}\left\{\int_{t}^{T} e^{-r(s-t)} G^{\varepsilon, \delta}\left(X_{s}^{\varepsilon}, Y_{s}^{\varepsilon}\right) d s\right\}\right| \leq c\{\varepsilon+\varepsilon|\log (\delta)|+\varepsilon \sqrt{\varepsilon / \delta}\} \\
\left|\mathbb{E}^{\star}{ }_{t, x, y}\left\{H^{\varepsilon, \delta}\left(X_{T}^{\varepsilon}, Y_{T}^{\varepsilon}\right)\right\}\right| \leq c\{\varepsilon+\varepsilon \sqrt{\varepsilon / \delta}\},
\end{gathered}
$$

and therefore also for $(t, x, y)$ fixed with $t<T$ :

$$
\begin{aligned}
\left|P^{\varepsilon, \delta}-Q^{\varepsilon, \delta}\right| & =\left|\varepsilon Q_{2}^{\delta}+\varepsilon^{3 / 2} Q_{3}^{\delta}-Z^{\varepsilon, \delta}\right| \\
& \leq c\{\varepsilon+\varepsilon|\log (\delta)|+\varepsilon \sqrt{\varepsilon / \delta}\} .
\end{aligned}
$$

since $Q_{2}^{\delta}$ and $Q_{3}^{\delta}$ evaluated for $t<T$ can also be bounded using (5.3) and (A.5). 
6. Conclusion. We have shown that the singular perturbation analysis of fast mean-reverting stochastic volatility pricing PDE's can be rigorously carried out for call options. We found that the leading order term and the first correction in the formal expansion are correct. The accuracy is pointwise in time, stock price and volatility level. It is precisely given in Theorem 3.1. The first correction involves higher order derivatives of the Black-Scholes price which blow up at maturity time and at the strike price. To overcome this difficulty we have used a payoff smoothing method and we have exploited the fact that the perturbation is around the Black-Scholes price for which there is an explicit formula. The case of call options is particularly important since the calibration of models is based on these instruments. The case of other types of singularities is open. With some work one can certainly generalize the method presented here to other European derivatives such as binary options. The case of path-dependent derivatives such as barrier options is more difficult due to the lack of an explicit formula for the correction. The situation with American contracts such as the simplest one, the American put, is much more involved due to the singularities at the exercise boundary.

\section{Appendices}

Appendix A. Expressions for Source Term and Terminal Condition. From (5.2), the source term in the equation for the error $Z^{\varepsilon, \delta}$ is

$$
G^{\varepsilon, \delta}=\varepsilon\left(\mathcal{L}_{1} Q_{3}^{\delta}+\mathcal{L}_{2} Q_{2}^{\delta}\right)+\varepsilon^{3 / 2} \mathcal{L}_{2} Q_{3}^{\delta} .
$$

To obtain an explicit form for this source term, we consider the three terms separately. We first introduce the convenient notation:

$$
\begin{aligned}
\mathcal{D} & \equiv \frac{\partial}{\partial x} \\
\mathcal{D}_{2} & \equiv \frac{\partial^{2}}{\partial x^{2}}-\frac{\partial}{\partial x} .
\end{aligned}
$$

Consider the term $\mathcal{L}_{2} Q_{2}^{\delta}$ in (A.1). Using that

$$
\begin{aligned}
\mathcal{L}_{2} & =\mathcal{L}_{B S}(f(y))=\mathcal{L}_{B S}(\bar{\sigma})+\frac{1}{2}\left(f(y)^{2}-\bar{\sigma}^{2}\right) \mathcal{D}_{2} \\
\mathcal{L}_{B S}(\bar{\sigma}) \mathcal{D}_{2} P_{0}^{\delta} & =0
\end{aligned}
$$

and (5.3), one deduces:

$$
\mathcal{L}_{2} Q_{2}^{\delta}=-\frac{1}{4}\left(f(y)^{2}-\bar{\sigma}^{2}\right) \phi(y) \mathcal{D}_{2} \mathcal{D}_{2} P_{0}^{\delta} .
$$

Consider next the term $\mathcal{L}_{1} Q_{3}^{\delta}$ in (A.1). Using (3.8) we have

$$
\begin{aligned}
Q_{3}^{\delta} & =-\mathcal{L}_{0}^{-1}\left(\mathcal{L}_{1} Q_{2}^{\delta}+\mathcal{L}_{2} P_{1}^{\delta}-\left\langle\mathcal{L}_{1} Q_{2}^{\delta}+\mathcal{L}_{2} P_{1}^{\delta}\right\rangle\right) \\
& =-\mathcal{L}_{0}^{-1}\left(\mathcal{L}_{1} Q_{2}^{\delta}-\left\langle\mathcal{L}_{1} Q_{2}^{\delta}\right\rangle+\left(\mathcal{L}_{2}-\left\langle\mathcal{L}_{2}\right\rangle\right) P_{1}^{\delta}\right)
\end{aligned}
$$

It follows from (5.3) that:

$$
\begin{aligned}
\mathcal{L}_{1} Q_{2}^{\delta} & =\left(\sqrt{2} \nu \rho f(y) \frac{\partial^{2}}{\partial x \partial y}-\sqrt{2} \nu \Lambda(y) \frac{\partial}{\partial y}\right)\left(-\frac{1}{2} \phi(y) \mathcal{D}_{2} P_{0}^{\delta}\right) \\
& =-\frac{1}{\sqrt{2}} \nu \rho f(y) \phi^{\prime}(y) \mathcal{D D}_{2} P_{0}^{\delta}+\frac{1}{\sqrt{2}} \nu \Lambda(y) \phi^{\prime}(y) \mathcal{D}_{2} P_{0}^{\delta}
\end{aligned}
$$


Now let $\psi_{1}$ and $\psi_{2}$ be solutions of

$$
\begin{aligned}
& \mathcal{L}_{0} \psi_{1}=f(y) \phi^{\prime}(y)-\left\langle f \phi^{\prime}\right\rangle, \\
& \mathcal{L}_{0} \psi_{2}=\Lambda(y) \phi^{\prime}(y)-\left\langle\Lambda \phi^{\prime}\right\rangle,
\end{aligned}
$$

then we find using (3.11) and (A.2) that $Q_{3}^{\delta}$ can be written:

$$
Q_{3}^{\delta}=\left(\frac{\nu \rho}{\sqrt{2}} \psi_{1}(y) \mathcal{D} \mathcal{D}_{2} P_{0}^{\delta}-\frac{\nu}{\sqrt{2}} \psi_{2}(y) \mathcal{D}_{2} P_{0}^{\delta}\right)-\frac{1}{2}\left(\phi(y) \mathcal{D}_{2} P_{1}^{\delta}\right)
$$

Substituting for $\mathcal{L}_{1}$ and expanding gives

$$
\begin{aligned}
\mathcal{L}_{1} Q_{3}^{\delta}= & \nu^{2} \rho^{2} f(y) \psi_{1}^{\prime}(y) \mathcal{D} \mathcal{D} \mathcal{D}_{2} P_{0}^{\delta}-\nu^{2} \rho f(y) \psi_{2}^{\prime}(y) \mathcal{D D}_{2} P_{0}^{\delta} \\
& -\nu^{2} \rho \Lambda(y) \psi_{1}^{\prime}(y) \mathcal{D D}_{2} P_{0}^{\delta}+\nu^{2} \Lambda(y) \psi_{2}^{\prime}(y) \mathcal{D}_{2} P_{0}^{\delta} \\
& -\frac{\nu}{\sqrt{2}}\left(\rho f(y) \phi^{\prime}(y) \mathcal{D} \mathcal{D}_{2} P_{1}^{\delta}-\Lambda(y) \phi^{\prime}(y) \mathcal{D}_{2} P_{1}^{\delta}\right) .
\end{aligned}
$$

Consider finally the term $\mathcal{L}_{2} Q_{3}^{\delta}$ in (A.1), we find using (A.2) and (A.5)

$$
\begin{aligned}
\mathcal{L}_{2} Q_{3}^{\delta}= & \frac{1}{2}\left(f(y)^{2}-\bar{\sigma}^{2}\right)\left[\frac{\rho \nu}{\sqrt{2}} \psi_{1}(y) \mathcal{D}_{2} \mathcal{D} \mathcal{D}_{2} P_{0}^{\delta}-\frac{\nu}{\sqrt{2}} \psi_{2}(y) \mathcal{D}_{2} \mathcal{D}_{2} P_{0}^{\delta}-\frac{1}{2} \phi(y) \mathcal{D}_{2} \mathcal{D}_{2} P_{1}^{\delta}\right] \\
& -\frac{1}{2} \phi(y) \mathcal{D}_{2}\left(v_{3} \mathcal{D}_{3} P_{0}^{\delta}+v_{2} \mathcal{D}_{2} P_{0}^{\delta}\right)
\end{aligned}
$$

with

$$
\mathcal{D}_{3}=\frac{\partial^{3}}{\partial x^{3}}-3 \frac{\partial^{2}}{\partial x^{2}}+2 \frac{\partial}{\partial x}
$$

and $v_{2,3}$ defined in (3.10).

To summarize, the source term is given by

$$
\begin{aligned}
G^{\varepsilon, \delta}= & \varepsilon\left\{\nu^{2} \rho^{2} f(y) \psi_{1}^{\prime}(y) \mathcal{D D} \mathcal{D}_{2} P_{0}^{\delta}-\nu^{2} \rho f(y) \psi_{2}^{\prime}(y) \mathcal{D} \mathcal{D}_{2} P_{0}^{\delta}\right. \\
& -\nu^{2} \rho \Lambda(y) \psi_{1}^{\prime}(y) \mathcal{D D}_{2} P_{0}^{\delta}+\nu^{2} \Lambda(y) \psi_{2}^{\prime}(y) \mathcal{D}_{2} P_{0}^{\delta} \\
& -\frac{\nu}{\sqrt{2}}\left(\rho f(y) \phi^{\prime}(y) \mathcal{D} \mathcal{D}_{2} P_{1}^{\delta}-\Lambda(y) \phi^{\prime}(y) \mathcal{D}_{2} P_{1}^{\delta}\right) \\
& \left.-\frac{1}{4}\left(f(y)^{2}-\bar{\sigma}^{2}\right) \phi(y) \mathcal{D}_{2} \mathcal{D}_{2} P_{0}^{\delta}\right\} \\
& +\varepsilon^{3 / 2}\left\{\frac{1}{2}\left(f(y)^{2}-\bar{\sigma}^{2}\right)\left[\frac{\rho \nu}{\sqrt{2}} \psi_{1}(y) \mathcal{D}_{2} \mathcal{D} \mathcal{D}_{2} P_{0}^{\delta}-\frac{\nu}{\sqrt{2}} \psi_{2}(y) \mathcal{D}_{2} \mathcal{D}_{2} P_{0}^{\delta}-\frac{1}{2} \phi(y) \mathcal{D}_{2} \mathcal{D}_{2} P_{1}^{\delta}\right]\right. \\
& \left.-\frac{1}{2} \phi(y) \mathcal{D}_{2}\left(v_{3} \mathcal{D}_{3} P_{0}^{\delta}+v_{2} \mathcal{D}_{2} P_{0}^{\delta}\right)\right\}
\end{aligned}
$$

By inspection, this can be written in the form (5.6).

From (5.3) and (A.5) we can also see that the terminal condition $H^{\varepsilon, \delta}$ in (5.5) can be written in the form (5.7).

Appendix B. Proof of Lemma 5.2. To prove Lemma 5.2 notice first that a calculation based on the analytic expression for the Black-Scholes price in the standard constant volatility case gives

$$
\partial_{x}^{n} P_{0}^{\delta}(s, x)= \begin{cases}e^{x} N(u / \tau+b \tau) & \text { for } n=1 \\ e^{x} N(u / \tau+b \tau)+\sum_{i=0}^{n-2} \frac{b_{i}^{(n)}}{\tau} e^{u} \partial_{u}^{i} e^{-(u / \tau+b \tau)^{2} / 2} & \text { for } n \geq 2\end{cases}
$$

for some constants $b_{i}$ and with

$$
\begin{aligned}
\tau & \equiv \bar{\sigma} \sqrt{T+\delta-s} \\
u & \equiv x-\log (K) \\
b & \equiv\left(r / \bar{\sigma}^{2}+1 / 2\right) .
\end{aligned}
$$


Assume first that $T-s \geq(T-t) / 2>0$, so that $\tau \geq \bar{\sigma} \sqrt{(T-t) / 2}$. Since $\partial_{x}^{i} P_{0}^{\delta}(s, x)$ is uniformly bounded in $\delta$, it follows that

$$
\left|\mathbb{E}_{t, x, y}^{\star}\left\{\chi\left(Y_{s}^{\varepsilon}\right) \partial_{x}^{i} P_{0}^{\delta}\left(s, X_{s}^{\varepsilon}\right)\right\}\right| \leq c \mathbb{E}^{\star} t, x, y\left\{\left|\chi\left(Y_{s}^{\varepsilon}\right)\right|\right\}
$$

for some constant $c$ which may depend on $x$.

Consider next the case $0<T-s<(T-t) / 2$, then

$$
\left|\mathbb{E}^{\star}{ }_{t, x, y}\left\{\chi\left(Y_{s}^{\varepsilon}\right) \partial_{x}^{i} P_{0}^{\delta}\left(s, X_{s}^{\varepsilon}\right)\right\}\right|=\left|\mathbb{E}^{\star}{ }_{t, x, y}\left\{\chi\left(Y_{s}^{\varepsilon}\right) \mathbb{E}^{\star}{ }_{t, x, y}\left\{\partial_{x}^{i} P_{0}^{\delta}\left(s, X_{s}^{\varepsilon}\right) \mid \hat{Z}_{v}^{\star} ; t \leq v \leq s\right\}\right\}\right|,
$$

and

$$
\begin{aligned}
& \left|\mathbb{E}^{\star}{ }_{t, x, y}\left\{\frac{1}{\tau} e^{u} \partial_{u}^{i} e^{-(u / \tau+b \tau)^{2} / 2} \mid \hat{Z}_{v}^{\star} ; t \leq v \leq s\right\}\right| \\
& =\frac{1}{\tau}\left|\int e^{u} \partial_{u}^{i} e^{-(u / \tau+b \tau)^{2} / 2} p(u) d u\right| \\
& =\frac{1}{\tau^{i}}\left|\int e^{\tau u} \partial_{u}^{i} e^{-(u+b \tau)^{2} / 2} p(\tau u) d u\right| \leq \frac{c}{\tau^{i}}
\end{aligned}
$$

where $p$ is the conditional distribution of $u \equiv X_{s}^{\varepsilon}-\log (K)$, which is the Gaussian distribution with variance at least $(T-t)\left(1-\rho^{2}\right) m_{1}^{2} / 2$. The bound (5.8) follows readily from (B.1), (B.2) and (B.3). The bound (5.9) is a direct consequence of (5.8) and Lemma 5.2 is established.

\section{Appendix C. On the solution of the Poisson equation.}

Let $\chi$ solve

$$
\mathcal{L}_{0} \chi+g=0
$$

with $\mathcal{L}_{0}$ defined as in (2.5) and with $g$ satisfying the centering condition

$$
\langle g\rangle=0,
$$

where the averaging is done with respect to the invariant distribution associated with the infinitesimal generator $\mathcal{L}_{0}$ (see (3.3) for an explicit formula). Using the explicit form of the differential operator $\mathcal{L}_{0}$, one can easily deduce that

$$
\Phi(y) \chi^{\prime}(y)=\frac{-1}{\nu^{2}} \int_{-\infty}^{y} g(z) \Phi(z) d z=\frac{1}{\nu^{2}} \int_{y}^{\infty} g(z) \Phi(z) d z
$$

with $\Phi$ being the probability density of the invariant distribution $\mathcal{N}\left(m, \nu^{2}\right)$ associated with $\mathcal{L}_{0}$. From this it follows that if $g$ is bounded

$$
\begin{aligned}
\left|\chi^{\prime}(y)\right| & \leq c_{1} \\
|\chi(y)| & \leq c_{2}(1+\log (1+|y|)) .
\end{aligned}
$$

Notice that $\chi$ in Lemma 5.1 satisfies

$$
|\chi(y)| \leq c \max \left(|\phi(y)|,\left|\phi^{\prime}(y)\right|,\left|\psi_{1,2}(y)\right|,\left|\psi_{1,2}^{\prime}(y)\right|\right)
$$

for some constant $c$ and with $\phi$ and $\psi_{1,2}$ defined in (3.11) and (A.4) respectively. These functions are solutions of Poisson equations with $g=f^{2}-\left\langle f^{2}\right\rangle$ or $g=f \phi^{\prime}-\left\langle f \phi^{\prime}\right\rangle$ or $g=\Lambda \phi^{\prime}-\left\langle\Lambda \phi^{\prime}\right\rangle$ which are bounded. Therefore $\chi(y)$ is at most logarithmically growing at infinity. The bound in Lemma 5.1 now follows from classical a priori estimates on the moments of the process $Y_{t}^{\varepsilon}$ which are uniform in $\varepsilon$. In the case $\Lambda=0$ this can easily be seen by a simple time change $t=\varepsilon t^{\prime}$ in (2.2). The case $\Lambda \neq 0$ follows by a Girsanov change of measure argument.

\section{REFERENCES}


[1] D. Duffie. Dynamic Asset Pricing Theory. Princeton University Press, 2nd Edition, 1996.

[2] J.-P. Fouque, G. Papanicolaou, and K.R. Sircar. Derivatives in Financial Markets with Stochastic Volatility. Cambridge University Press, 2000.

[3] J.-P. Fouque, G. Papanicolaou and K.R. Sircar. Mean-reverting stochastic volatility. International J. Theor. and Appl. Finance, 13(2): 101-142. (2000).

[4] J.-P. Fouque and T. Tullie. Variance Reduction for Monte Carlo Simulation in a Stochastic Volatility Environment. Quantitative Finance 2: 24-30 (2002).

[5] R. Frey. Derivative asset analysis in models with level-dependent and stochastic volatility. CWI Quarterly 10(1), pp 1-34, 1996.

[6] E. Ghysels, A. Harvey and E. Renault. Stochastic volatility, in G. Maddala and C. Rao (eds), Statistical Methods in Finance, Vol. 14 of Handbook of Statistics, North Holland, Amsterdam, chapter 5, pp 119-191, 1996.

[7] J. Hull. and A. White. The Pricing of Options on Assets with Stochastic Volatilities. J. Finance XLII(2), pp 281-300, 1987.

[8] G. Willard. Calculating prices and sensitivities for path-independent derivative securities in multifactor models PhD thesis, Washington University in St. Louis, MO (1996). 